\title{
Zimt - nicht nur zur Weihnachtszeit
}

\section{Eine Übersicht zur Verwendung von Zimt als Gewvïrz und Heillmittel}

Cäcilia Brendieck-Worm

\section{๑) Zusammenfassung}

Zimt hat als Gewürz und Heilmittel eine jahrtausendealte Tradition. Seit sein antidiabetogenes Potenzial entdeckt wurde, steht Zimt im Fokus pharmakologischer Forschung und ist als mögliches Heilmittel gegen die weltweit expandierenden Volkskrankheiten Adipositas und Diabetes mellitus Typ 2 heute interessanter denn je. Die aktuelle Geschichte des Zimts zeigt exemplarisch die Problematik des heutigen Umgangs mit Pflanzenstoffen. So werden z. B. als Duftstoffe geeignete Einzelsubstanzen extrahiert, synthetisiert und breit verwendet, wie dies mit im Zimt vorkommendem Cumarin und Zimtaldehyd geschieht. Bei isolierten Stoffen verstärkt sich nicht selten die Tendenz zur Allergisierung, wie sie für Zimtaldehyd bekannt ist. Positive Erfahrungen mit der Verwendung einer Pflanzenzubereitung als Gewürz garantiert zudem nicht zwangsläufig die Unbedenklichkeit einer deutlich höheren täglichen Langzeiteinnahme als Nahrungsergänzung. Es ist weitere Forschung zu Nutzen und Risiken therapeutischer Zimtanwendung nötig.

\section{Einleitung}

Zimt wird seit Jahrtausenden aufgrund seiner geschmacklichen und verdauungsfördernden Qualitäten in großen Teilen der Welt geschätzt und vielfältig genutzt.

Unsicherheit in Bezug auf die Unbedenklichkeit von Zimt tauchte hierzulande erst 2006 auf, als das Bundesamt für Risikobewertung (BfR) vor Cumarin im Zimt warnte [1] (s. Kasten Gefährliche Zimtsterne?).

Cumarine sollen lebertoxisch und karzinogen wirken, zumindest legen dies Versuche mit extrem hohen Dosen bei Ratten nahe. Hinweise auf Schäden beim Men-

\begin{abstract}
Gefährliche Zimtsterne?
Als man in zimthaltigem Weihnachtsgebäck Cumarin-Werte gemessen hatte, die den in der Aromenrichtlinie vorgegebenen Höchstwert von $2 \mathrm{mg} / \mathrm{kg}$ erheblich überschritten, sah sich das BfR genötigt, darauf hinzuweisen, dass Kinder, die vielleicht zusätzlich noch Milchreis mit Zucker und Zimt verzehrten, im worst-case-Szenario mit einer Cumarinmenge belastet werden könnten, die den TDI (tolerable daily intake) und damit die Menge, die täglich ein Leben lang ohne Gesundheitsschäden aufgenommen werden kann, überschreitet. 2012 kam das BfR in einer Neubewertung zu denselben Schlüssen [3].
\end{abstract}

schen und beim Haustier durch Cumarin oder cumarinhaltigen Zimt fehlen bislang (s. Kasten Cumarin). Während die Gefahr durch weihnachtliche Zimtsterne wohl eher anekdotischen Charakter hat, könnte der Trend, Zimt im Grammbereich in der täglichen Daueranwendung zur Blutzuckersenkung einzunehmen, schon eher als bedenklich angesehen werden [1,2]. Doch nicht jeder Zimt enthält überhaupt Cumarine. Außerdem könnte auch ein weiterer Inhaltsstoff im Zimt für Probleme sorgen: das Zimtaldehyd. Auch Zimtaldehyd kommt nicht in jeder Zimtzubereitung vor (s. Kasten Zimtaldehyd). Die folgenden Ausführungen sollen zur Klarheit über den Zimt beitragen.

\section{Geschichte des Zimts}

Zimt wird bereits im Kräuterbuch des chinesischen Kaisers Shen Nung (ca. 2700 v. Chr.) als hoch geschätzte Zutat der chinesischen Küche erwähnt. Chinesische Heilkundige beschrieben schon früh seine Yang-tonisierende Wirkung (s.u., Zimt in der TCM).

\begin{abstract}
Cumarin
Cumarin ist das Lacton der cis-o-Hydroxyzimtsäure. Seinen charakteristischen Geruch verbindet man mit frischem Heu und Waldmeisterbowle. Cumarin wirkt sedativ, spasmolytisch, antiphlogistisch, ödemhemmend/ antiexsudativ und lymphokinetisch. Ihm wird eine antitumorale, insbesondere rezidiv- und metastasenhemmende Wirkung über direkte Immunstimulation zugesprochen. Cumarin hemmt nicht die Blutgerinnung, wie fälschlich vielfach behauptet wird (blutgerinnungshemmend wirkt Dicumarol).

Cumarin wird beim Menschen nach peroraler Aufnahme durch CYP2A6, einen Subtyp des Cytochrom P450, hauptsächlich zu 7-Hydroxycumarin umgewandelt und verlässt den Körper als Glucuronid oder Sulfat über den Urin. Geringe Anteile werden zu lebertoxischem Cumarin-3,4-epoxid metabolisiert, das durch Glutathion entgiftet wird.

Bei vorgeschädigter Leber oder einem genetischen Defekt im Entgiftungssystem sind hohe tägliche Cumarindosen möglicherweise gefährlich. Aus Sicherheitsgründen wurde deshalb für den Menschen eine tolerierbare tägliche Einnahmemenge (TDI) von $0,1 \mathrm{mg} / \mathrm{kg}$ festgelegt, was bei einem $60 \mathrm{~kg}$ schweren Menschen lebenslang $6 \mathrm{mg} / \mathrm{Tag}$ entspräche. Die Ratte - das Standard-Labortier zur Gewinnung toxikologischer Daten - ist aufgrund eines anderen Metabolisierungsweges durch Cumarin wesentlich stärker gefährdet als z. B. der Mensch. Auch für Katzen muss aufgrund der Glucuronidierungsschwäche durch tägliche Cumarinzufuhr mit einer Gesundheitsgefährdung gerechnet werden. Cumarin findet als Duftstoff Verwendung in der Parfumerie und wird auch über die Haut aufgenommen $[1,2,14]$.
\end{abstract}

Bereits vor 4000 Jahren war Zimt ein wichtiges Handelsprodukt zwischen China und Indien. Über die Seidenstraße, ein Netz von Karawanenrouten, das Ostasien mit dem mediterranen Raum verband, gelangte Zimt nach Mittelasien und Osteuropa. Zimt findet sich in der Bibel, so im Buch Mose, als Bestandteil des heiligen 
Zimtaldehyd - eine Substanz mit allergenem Potenzial

Zimtaldehyd (trans-Zimtaldehyd) ist wesentlicher Bestandteil in ätherischem Zimtöl. Es wird jedoch v. a. synthetisch hergestellt und findet als Duftstoff in Kosmetika, Wasch- und Reinigungsmitteln, Zahncremes und Mundwässern große Verbreitung. Zimtaldehyd kann über die Haut aufgenommen werden und ist beim Menschen recht häufig allergieauslösend auf Haut und Schleimhaut (Allergien vom Typ IV). Zimtaldehyd kommt in wässrigen Zimtextrakten nicht vor $[11,18]$.

Salböls. Im Papyrus Ebers, einer medizinischen Schrift aus dem alten Ägypten (16.Jh.v. Chr.) wird Zimt als Ingredienz des berühmten Kyphi aufgeführt, einer kostbaren Räuchermischung, die man den Göttern darbot. Im griechischen und römischen Reich wurde Zimt v.a. wegen seiner verdauungsfördernden und harntreibenden Wirkung, aber auch aufgrund seiner anregenden und konservierenden (antiseptischen) Eigenschaften geschätzt. Ins westliche Europa gelangte Zimt in großen Mengen erst nach der Entdeckung des Seewegs nach Indien durch die Portugiesen im 15. Jahrhundert. Dieser Seeweg wurde auch „Gewürzroute“ genannt, waren es doch gerade die Gewürze wie Zimt, Pfeffer, Nelken und Muskat, die den Eroberern zu großem Reichtum verhalfen. Denn diese Gewürze boten nicht nur kulinarischen Genuss, sondern waren als Heilund Konservierungsmittel von unschätzbarem Wert. Nach der Besitznahme der Insel Ceylon (heute Sri Lanka) durch die Portugiesen hielten diese das Handelsmonopol für Zimt, das ihnen erst 1658 die Niederländer streitig machten, die schließlich zum Ende des 18. Jahrhunderts von Großbritannien aus ihrer lukrativen Position verdrängt wurden. Anfangs wurden in Ceylon nur wilde Bäume zur Drogengewinnung genutzt. Die ersten Zimtkulturen gehen auf die Initiative der Holländer zurück. Bis 1840 war Zimt der Hauptausfuhrartikel Ceylons $[8,13,15]$.

\section{Botanik}

Beim Zimt handelt es sich um die Rinde von Bäumen der Gattung Cinnamomum aus der Familie der Lorbeergewächse (Lauraceae), die sich über die tropischen und

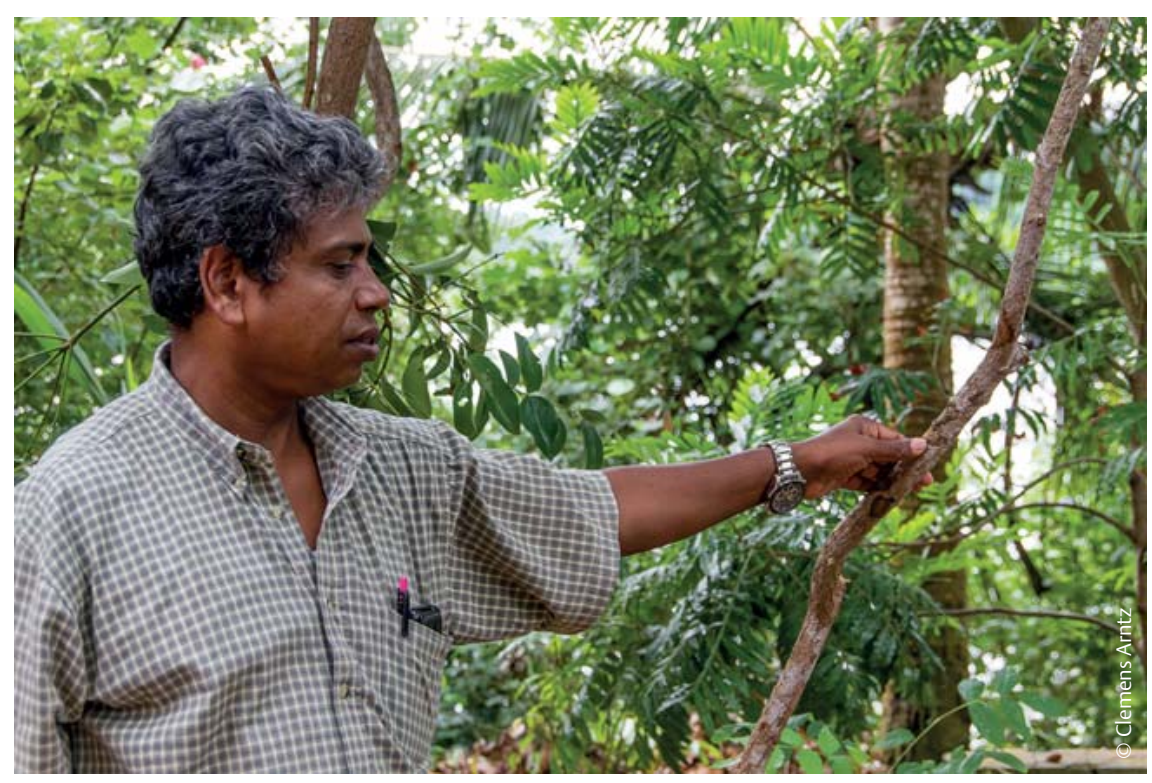

Abb. 1 Zur Zimtgewinnung werden Stockausschläge geschält.

subtropischen Regionen Asiens, Australiens, der Pazifischen Inseln sowie Südamerikas verteilen [6].

\section{Cinnamomum verum, der Echte Zimt}

Als echter, wertvollster und teuerster Zimt galt stets die Rinde des Ceylonischen Zimtbaumes (Kaneelbaum), Cinnamomum verum J.S. PRESL (syn. Cinnamomum ceylanicum, Laurus cinnamifera). Die Heimat des Ceylonischen Zimtbaumes sind die Bergwälder Südwestindiens und im Westen Sri Lankas. Von dort gelangte er in viele andere tropische Regionen: nach China, auf die Komoren, die Seychellen und die Westindischen Inseln, nach Java, Madagaskar und Südamerika [10,13].

\section{Cinnamomum aromaticum, der Chine- sische Zimt}

Wirtschaftlich große Bedeutung hat zudem der Chinesische Zimt, auch bezeichnet als Kassia-Zimt, Gemeiner Zimt, Holzzimt, Mutterzimt u.a., gewonnen vom Chinesischen Zimtbaum (Zimtkassia), Cinnamomum aromaticum NEES, syn. Cinnamomum cassia PRESL. Chinesischer Zimt stammt aus dem Süden von China, aus Myanmar, Laos, Kambodscha und Vietnam, wo noch heute seine Hauptanbaugebiete liegen. Weitere Kulturen finden sich in Indonesien, Sri Lanka und Südamerika.

Der Echte und der Chinesische Zimtbaum gehören zu den immergrünen Bäumen und werden ca. $18 \mathrm{~m}$ hoch. Blätter, Blüten und Früchte unterscheiden sich nur geringfügig. In Kulturen werden die Stämme regelmäßig gestutzt, um die Entwicklung von Stockausschlägen $\mathrm{zu}$ fördern, deren Rinde zur Zimtgewinnung geschält wird (\$Abb. 1).

Die sogenannten "Zimtstangen", die getrocknete Rinde der beiden Arten, lassen sich gut unterscheiden:

Typisch für C. verum ist eine in getrocknetem Zustand nur Bruchteile von Millimetern dicke, gelbbraune, blättrige, brüchige Rinde, geschält von 1-2-jährigen Stockausschlägen, von der die äußere Korkschicht und das innere Kambium abgelöst wurde (\$Abb. 2 und 3). Beim Trocknen rollen sich die ca. 2-4 m langen Rindenstücke seitlich ein. Sie werden auf ca. 75 cm gekürzt, jeweils zu 6-10 zu Rollen (Quills) ineinandergeschoben und als Ballen verschifft. Gehandelt werden sie als 8-10 cm lange Stücke (\$ Abb. 4). Beim Schneiden anfallende Reste werden zu Zimtpulver vermahlen oder es wird Zimtöl aus ihnen destilliert. Der Echte Zimt zeichnet sich durch milden, süßlichen Geruch und Geschmack aus.

Die grau-braunen Zimtstangen vom Chinesischen Zimt $C$. aromaticum sind mit 1-3 mm wesentlich dicker und unregelmäßiger, da zu ihrer Gewinnung bis zu 6 Jahre alte Stockausschläge geschält und deren Rinde nur unvollständig von der Korkschicht befreit werden (\$ Abb. 4). Die gehandelten Röhren sind bis $40 \mathrm{~cm}$ lang. Geruch und Geschmack sind im Vergleich mit C. verum deutlich herber $[6,10,13]$. 


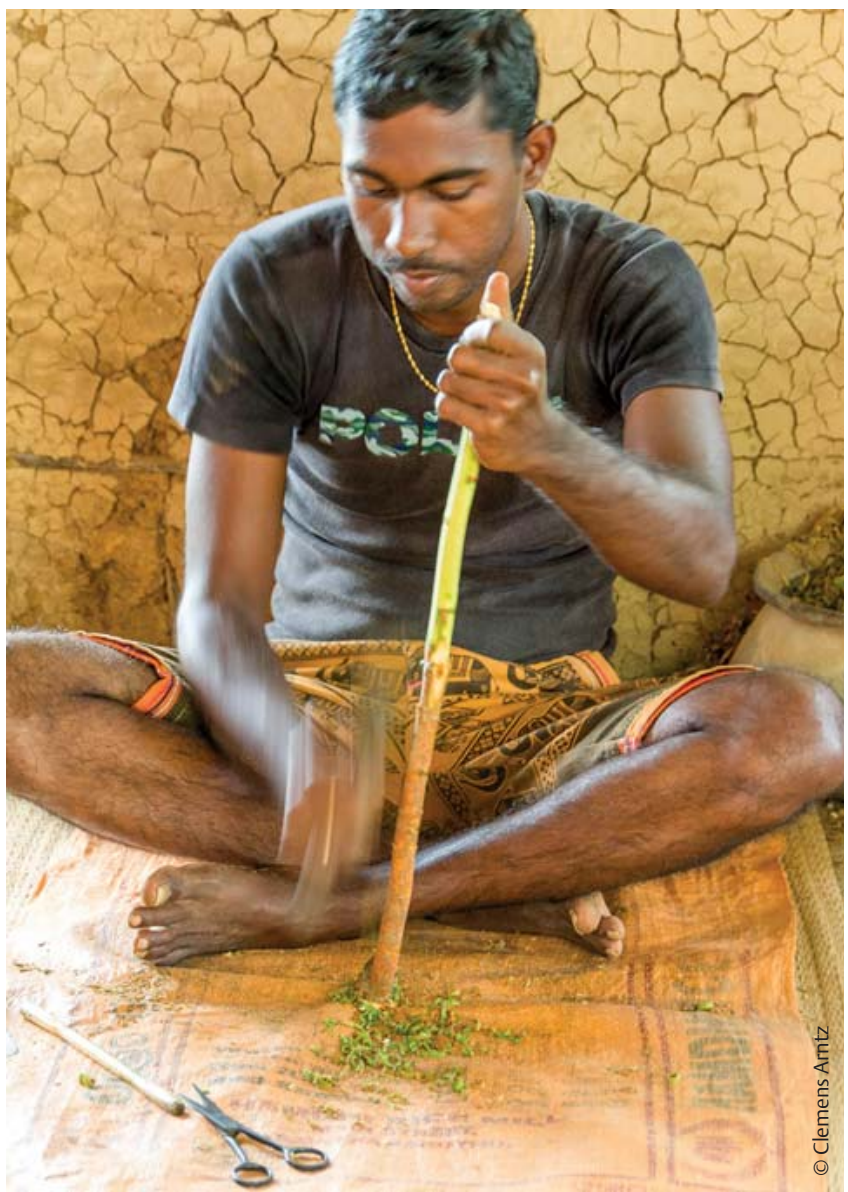

Abb. 2 Zuerst wird die äußere Korkschicht abgeschabt.

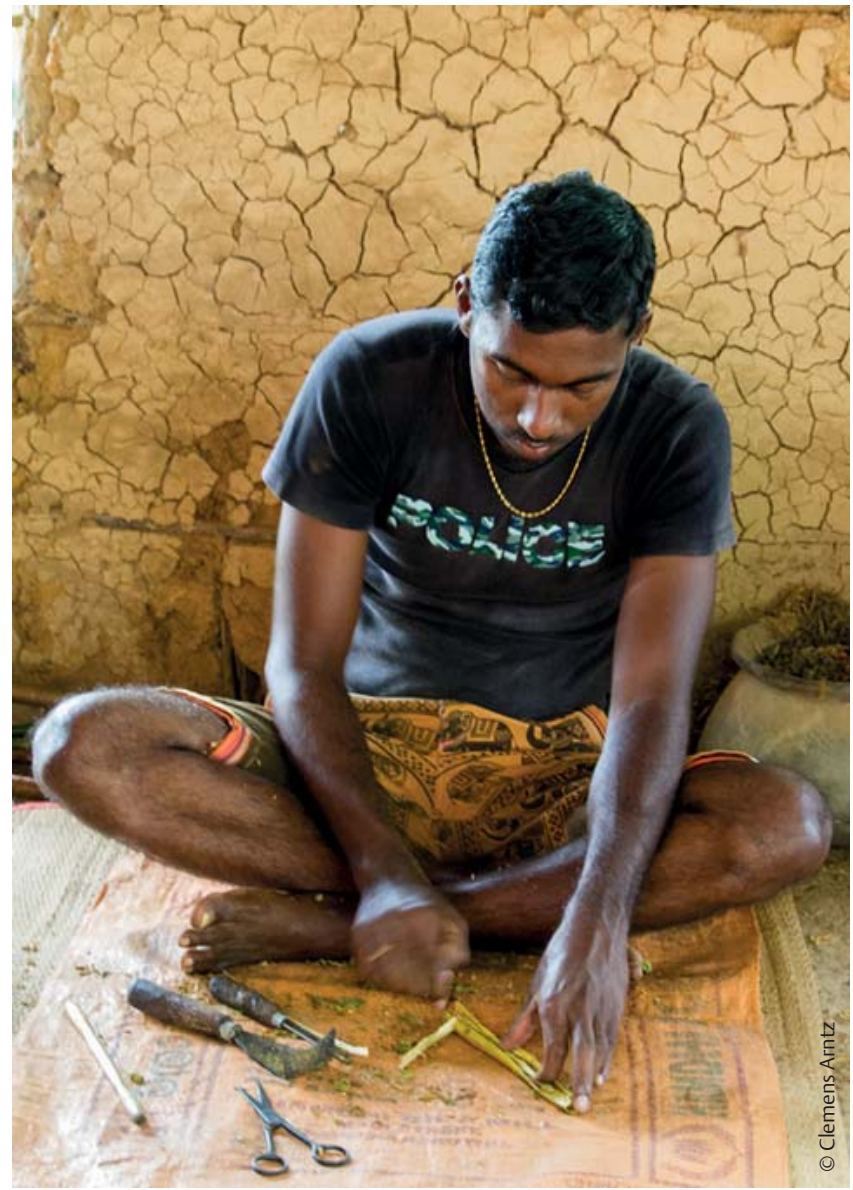

Abb. 3 Dann wird das innere Kambium abgelöst.

\section{Die Verwendung von Zimt}

Echter Zimt und Chinesischer Zimt werden sowohl als Gewürz als auch therapeutisch etwa gleich angewendet.

\section{Zimt als Gewürz}

In arabischen Ländern und Südostasien ist Zimt ein wichtiges Gewürz. Es gehört in die arabische Gewürzmischung Baharat, mit der man Fleisch- und Fischgerichte, aber auch den Mokka würzt. Zimt kommt in Schmorgerichte wie die marokkanische Tajine, den iranischen Khorak, in die herzhaften Reisgerichte Pilaw (Polow) und Biryani, ist wichtige Zutat in asiatischen Kormas und Currys.

In der westlichen Welt gehören Zucker und Zimt zusammen. Man nutzt Zimt insbesondere zum Würzen von Süßspeisen und Gebäck wie Lebkuchen, Spekulatius und Pfannkuchen. Beliebt ist auch das Würzen von Heißgetränken wie Glühwein, Punsch, Kakao und Kaffee. Auch Cola enthält Zimt [13].

\section{Zimt als Therapeutikum}

\section{Echter Zimt}

In das Europäische Arzneibuch ist bisher lediglich Cinnamomum verum aufgenommen worden.

Therapeutisch genutzt werden Zimtrinde und ätherisches Öl aus Rinde und Blättern:

1. Zimtrinde (syn. Ceylonzimtrinde, Echter Kaneel, Echter Zimt, Java-Zimt, MalabarZimt)/Cinnamomi cortex (syn. Cinnamomi ceylanici cortex, Cortex Cinnamomi, Cortex Cinnamomi ceylanici)

\section{Inhaltsstoffe:}

a ätherisches Öl (0,5-2,5\%), v.a. mit Zimtaldehyd (ca. 75\%), Eugenol, Linalool, $\beta$-Caryophyllen

- Gerbstoffe, v.a. oligomere Procyanidine

Schleimstoffe (2-4\%)

- Diterpene

Phenolcarbonsäuren

\section{Wirkungen:}

antimikrobiell (antibakteriell, fungistatisch)

antiphlogistisch

- spasmolytisch

arminativ

motilitätsfördernd

- Steigerung der Speichel- u. Magensaftsekretion (Hyperämisierung der Schleimhäute)

antidiabetisch (Senkung des Blutzuckerspiegels, verstärkte Insulinaktivität, Senkung des LDL-Cholesterins)

Indikationen nach ESCOP-, WHO- und HMPC-Monografie:

innerlich (Tee, Tinktur)

- Appetitlosigkeit

dyspeptische Beschwerden, wie Darmspasmen, Flatulenz, Völlegefühl

- Bauchschmerzen mit Diarrhö

Schmerzen bei Amenorrhö, Dysmenorrhö 


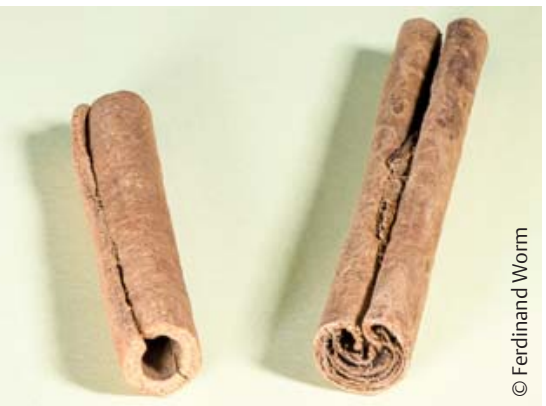

Abb. 4 Chinesische Zimtrinde (li.) ist mit 1-3 mm wesentlich dicker als Echte Zimtrinde (re.), von der jeweils mehrere Stücke ineinander geschoben werden.

\section{volksheilkundliche Anwendung:}

innerlich (Tee, Tinktur)

zur Verdauungsförderung

bei leichten gastrointestinalen Spasmen

bei Dysmenorrhö

als Hämostyptikum

als begleitende Diät bei Diabetes mellitus

\section{Kontraindikationen Mensch:}

Überempfindlichkeit gegenüber Zimt, Tolu- und Perubalsam (Kreuzreaktionen), Schwangerschaft (verstärkt Uteruskontraktionen, Abortgefahr)

Nicht bei Atopikern einsetzen, Ausnahme wässrige Extrakte (Zimtaldehyd-frei). Nicht einsetzen bei Verdacht auf MagenDarmgeschwüre.

Nebenwirkungen Mensch:

häufig allergische Haut- und Schleimhautreaktionen ( $\rightarrow$ Kasten: Zimtaldehyd)

\section{Zimtrindenöl $[8,15]$}

ätherisches Öl aus der Rinde, Cinnamomi ceylanici corticis aetheroleum, syn. Aetheroleum cinnamomi; Inhaltsstoffe: s.o.

\section{Wirkungen:}

antimikrobiell (antibakteriell, fungizid, antiviral)

analgetisch

spasmolytisch

karminativ

motilitätsfördernd

hyperämisierend (Steigerung der Speichel- u. Magensaftsekretion, Durchwärmung von Muskulatur)

tonisierend, sympathikoton

\section{Indikationen:}

äußerlich (Auflagen, Massagen, Bäder)

Diarrhö, Enterocolitis, Dysenterie

- Zystitis

- Bronchitis, Pleuritis

- Amenorrhö, Dysmenorrhö, Geburtseinleitung und Wehenauslösung (Suppositorien)

- Muskel- und Gelenkschmerzen, Arthritis

3. Zimtblätteröl [15], ätherisches Öl aus den Zimtblättern, Cinnamomi ceylanici folii aetheroleum

Inhaltsstoffe:

Hauptbestandteil: Eugenol, weniger Zimtaldehyd (dadurch weniger haut- und schleimhautreizend/allergisierend),

(große Ähnlichkeit mit Nelkenöl).

\section{Wirkungen:}

- stark bakterizid

antiviral

- fungizid

- antiparasitär

analgetisch

- tonisierend

- immunstimulierend (IgA $\uparrow$ )

\section{Indikationen:}

äußerlich (Auflagen, Massagen, Bäder, Inhalation)

Enterocolitis

- Stomatitis

Zahnschmerzen

- Zystitis

- Bronchitis, Rhinopharyngitis

Geschwächtes Immunsystem

- Asthenie

\section{Chinesischer Zimt}

Chinesischer Zimt wird in der Volksmedizin analog zum Echten Zimt eingesetzt.

1. Chinesische Zimtrinde (syn. KassiaZimt, Englischer Zimt, Gemeiner Zimt, Mutterzimt, Indischer Zimt)/Cinnamomi chinensis cortex (syn. Cinnamomi cassiae cortex, Cortex Cinnamomi cassiae, Cortex Cinnamomi)

2. Zimtrindenöl, das ätherische Öl der Chinesischen Zimtrinde Cinnamomi cassiae aetheroleum, Kassiaöl, gewonnen aus Rindenabfällen, Blättern und jungen Zweigen.

3. Zimtblüten (syn. Kanelblüten, Kassiablüten, Zimtkelche, Zimtnägelein), Cassiae flos (syn. Cinnamomi flos, Clavellis Cassiae, Flores cassiae), die nach dem Verblühen gesammelten, getrockneten Blüten (eigentlich junges Fruchtstadium). Hauptkomponente der Inhaltsstoffe ist Zimtaldehyd. Angewendet wird die Droge in der Volksmedizin zur Blutreinigung und in Kombinationen gegen Entzündungen sowie als Geruchs- und Geschmackskorrigenz.

Der wesentliche Unterschied zum Echten Zimt besteht im Gehalt an Cumarin im ätherischen Öl, der durch die Art der Zimtrindengewinnung bedingt ist: Chinesische Zimtrinde wird nur unvollständig von der Korkschicht befreit, die das Cumarin enthält [11]. Je dicker die Rinde, umso höher die Ausbeute bei der Zimtrindengewinnung, umso mehr Cumarine, umso unempfindlicher aber auch das Produkt, umso günstiger der Preis. Werden in Fertigprodukten hohe Cumaringehalte beanstandet, so ist dies ein sicherer Hinweis auf die Verwendung des preiswerteren Chinesischen Zimts [1,2].

\section{Zimt in der Traditionellen Chinesischen Medizin (TCM)}

In der TCM dient Zimtrinde (chin. Rougui) als wärmende, stimulierende, adstringierende und entspannende Arznei. Sie wird eingesetzt als kräftigendes Herz- und Kreislaufstimulans, bei Folgen von YangMangel in Herz und Nieren, zur Förderung der Verdauung und zur Schmerzlinderung. Zimtzweige, syn. Kassia-Zimtzweige, Cinnamoni ramulus, (chin. Guizhi) finden Anwendung bei Folgen von Erkältungen, Schmerzen in Magen und Unterleib, Amenorrhö, Säftestau etc. [8,9].

\section{Zimt in der Veterinärmedizin}

Schon in der sogenannten Stallmeisterzeit (15.-18. Jahrhundert) findet man Zimt (Zimmet, Ziemet, Zimet Rinden) als vielfältig einzusetzendes Therapeutikum. In dem Rossarzneibuch des Joachim Christoph Zachen wird Zimt als schweißtreibend beschrieben und in Mischungen zur allgemeinen Herzstärkung und Unterstützung bei Krankheit empfohlen [7]. In der tiermedizinischen Fachliteratur zum Ende des 19. Jahrhunderts gilt Zimt als gewürzhaftes Stomachikum, Geschmackskorrigenz und krampfstillendes Mittel bei Uterus-, Darm- und Blasenkrämpfen. Auch 


\section{Traditionelle Zimtrezepturen [5]}

Eine Rezeptur zur Stärkung war „Stockes Brandy-egg Mixtur“

Rp. Sirupi Cinnamoni 25,0

Aqua Cinnamoni 100,0

Spiritus e vino 50,0

M.D. S. Mit 2 Eigelb verrührt esslöffelweise zu geben für an Staupe erkrankte Hunde. Gebräuchliche Zubereitungsformen waren:

Aqua Cinnamoni (Zimtwasser), durch Destillation von Zimt mit Wasser und Weingeist gewonnen

- Tinktura Cinnamomi (Zimttinktur), aus 1 Teil Zimt und 5 Teilen verdünntem Weingeist bereitet

- Syrupus Cinnamoni (Zimtsirup), Mazeration aus Zimt mit Zimtwasser und Zucker

- Tinctura aromatica: bereitet aus Zimt, Nelken, Ingwer, Cardamom, Galgant und verdünntem Weingeist

seine starke antiseptische Wirkung war bereits bekannt. Zimtöl wurde daher auch zur Händedesinfektion eingesetzt (10\%ig). Zimt galt als exzitierende (stimulierende) Arznei und wurde bei Schwächezuständen im Laufe von Geburten des Rindes mit Glühwein oder Warmbier verabreicht. Als Dosierung für Zimtpulver wurde empfohlen in g/Tag [5]:

- Pferd, Rind: 10-20

Schaf, Ziege, Schwein: 5-10

Hund: 0,2-1

Katze: 0,1-0,25

Zimt wird auch aktuell in der Tiermedizin als Ergänzungsfuttermittel in verschiedenen Kombinationen angeboten. Ein traditionelles Arzneimittel für Tiere (Pferde, Rinder, Schweine, Schafe, Ziegen, Kaninchen, Hunde) ist das ColoSan ${ }^{\circledR}$ (SaluVet $\mathrm{GmbH}$, Bad Waldsee), eine Lösung aus Chinesischem Zimtöl, Anisöl, Bitterfenchelöl, Kümmelöl und Schwefel, die sich bei Verdauungsproblemen mit Blähungen und Koliken bewährt hat.

\section{Zimt in der aktuellen Forschung}

Adipositas und Diabetes mellitus Typ 2

Der hauptsächlich durch Ernährungsfehler und Bewegungsmangel ausgelöste Diabetes mellitus Typ 2 gilt weltweit als großes gesundheitliches Problem. Die Therapie dieser chronischen metabolischen Störung mit ihren gefürchteten Spätfolgen wie Nephropathie, Retinopathie, Neuropathie und kardiovaskulären Schäden ist bisher unbefriedigend. Synthetische Antidiabetika zeigen häufig unerwünschte Nebenwirkungen. 1990 wurde erstmals über die positiven Wirkungen des Zimts bei Diabetes berichtet. 2003 konnte beim Menschen nachgewiesen werden, dass die tägliche Einnahme von Zimt (Cinnamomum verum) den Blutzuckerspiegel, Triglyceride, Gesamtcholesterol und LDL-Cholesterol senkt. Als wesentlich für die Wirkung werden die Zimtaldehyde betrachtet. Möglicherweise erhöhen sie die Expression des Glucosetransporters-4 (GLUT-4), der die Effektivität des Insulins steigert. Typ-A-Procyanidin wirkt zudem antioxidativ, schützt dadurch die $\beta$-Zellen des Pankreas und erhöht die Effektivität des Insulins. Typ-B-Procyanidin verbessert die Insulinsensitivität. Zimtrindenextrakte erwiesen sich zudem als Inhibitoren der $\alpha$ Amylase und der $\alpha$-Glucosidase. Nach derzeitigem Stand der Forschung gilt Cinnamomum aromaticum (syn. C. cassia) als effektivstes Antidiabetikum unter den Zimtarten [4]. Unter vielen auf ihre Wirksamkeit gegen Adipositas und Diabetes getesteten pflanzlichen Substanzen erwies sich der wässrige Zimtextrakt als potentester Inhibitor von Amylasen und Lipasen (enthält keine Zimtaldehyde!) [12].

\section{Arzneimittel oder Nahrungsergänzer}

Während in der Forschung noch nach Wirkmechanismen und optimalen Zubereitungen gesucht wird, vermarkten einige Hersteller von Nahrungsergänzern Zimt bereits als Diabeteskiller und Fatburner, zur Blutzucker- und Cholesterinsenkung, zur Krebs- und Alzheimerprävention. Dies ist eine kritisch zu betrachtende Tendenz. Wird doch unter Umständen dadurch suggeriert, bei Zimtkapseln handele es sich um die lange gesuchten Lifestyle-Pillen, mit denen man auf natürlichem Wege die Mängel in der Lebensführung wettmachen kann. Das Bundesinstitut für Arzneimittel und Medizinprodukte (BfArM) ist denn auch der Ansicht, dass es sich bei Zimtkapseln um ein Arzneimittel handelt, für das entsprechende Zulassungsbedingungen zu erbringen seien.

\section{Aus der Praxis (Yvonne Thoonsen)}

Ätherischöl-Zubereitung zur äußerlichen Anwendung bei Dermatophyten des Meerschweinchens:

Rp:

Sheabutter $\quad 50 \mathrm{ml}$

Kokosnussöl $\quad 50 \mathrm{ml}$

Nelkenknospenöl 8 Tropfen

Zimtblätteröl 25 Tropfen

Lemongrasöl 30 Tropfen

Salbe herstellen und täglich $1 \times$ dünn auf die betroffenen Stellen auftragen. Vorsicht im Bereich der Augen! Sehr gute fungizide und juckreizlindernde Wirkung.

Nach 14 bis spätestens 21 Tagen waren die Pilzinfektionen abgeklungen.

\section{Industrielle Tierproduktion - die Suche nach Ersatz für antibiotische Leistungsförderer}

Drogen mit ätherischen Ölen gehören zu den Futterzusatzstoffen, die sich leistungsfördernd und gesundheitsstärkend auswirken können. Sie steigern die Durchblutung, regen die Sekretion von Verdauungssekreten an, steigern die Resorption, reduzieren oxidativen Stress im Verdauungskanal und fördern die Immunkompetenz des Tieres. All das führt zu einer besseren Entwicklung, wie dies bei Schwein und Geflügel bereits belegt ist. Die Wirkmechanismen liegen jedoch noch weitgehend im Dunkeln. Insbesondere sind Interaktionen zwischen Nahrungsbestandteilen, der Darmflora und den ätherischen Ölen zu vermuten.

In der Geflügel- und Schweinemast ist insbesondere das Zimtaldehyd von Interesse, auch in Kombination mit Einzelkomponenten wie Carvacrol und Thymol, die für ihre antimikrobielle Aktivität bekannten Bestandteile aus den ätherischen Ölen von Thymian und Oreganum. Doch noch gibt es keine konkreten Daten zur Dosierung und zu synergistischen oder antagonistischen Effekten [17]. Auch in der Kälber- und Bullenmast wurde mit Zimt als Futterzusatz experimentiert und in frühen Mastphasen eine leicht verbesserte Futteraufnahme sowie weniger Stressanfälligkeit festgestellt [16].

Ob sich die antidiabetogene Wirkung des Zimts auch bei Adipositas und metabolischem Syndrom des Hundes therapeutisch nutzen lässt, ist noch zu klären. Anwendungserfahrungen sprechen dafür. $\odot$ 


\section{() Summary}

Cinnamon - Not Just for Christmas: An Overview of the Use of Cinnamon as a Spice and Herbal Remedy

Cinnamon has a millenary tradition as a spice and medicine. Since its antidiabetogenic potential was discovered, cinnamon has been in the focus of pharmacological research and has gained more importance as a possible remedy against the world's expanding common diseases of obesity and diabetes mellitus type 2 . The current history of cinnamon exemplifies the problems encountered while dealing with herbal extracts. A case in point concerns individual substances suitable for fragrances that are extracted, synthesized and widely used such as coumarin and cinnamaldehyde from cinnamon. Isolated substances like cinnamaldehyde often tend to lead to allergies. Thus the positive experience of the use of spices from herbal preparations does not necessarily guarantee their safety and their higher daily longterm use as dietary supplements. In this vein, it is necessary to carry out further research on the benefits and risks of the therapeutic application of cinnamon.

\section{(1) Key words}

Cinnamon - spice - herbal remedy - antidiabetogenic potential

\section{() Literatur}

[1] Bundesinstitut für Risikobewertung (BfR). Gesundheitliche Bewertung des BfR Nr. 043/2006 vom 16. Juni 2006. http://www.bfr.bund.de/ cm/343/verbraucher_die_viel_zimt_ verzehren_sind_derzeit_zu_hoch_mit_ cumarin_belastet.pdf

[2] Bundesinstitut für Risikobewertung (BfR). Gesundheitliche Bewertung des BfR, Nr. 044/ 2006 vom 18. August 2006. http://www.bfr. bund.de/cm/343/hohe_taegliche_ aufnahmemengen_von_zimt_ gesundheitsrisiko_kann_nicht_ ausgeschlossen_werden.pdf

[3] Bundesinstitut für Risikobewertung (BfR). Presseinformation 26/2012, 27.09.2012. http://www.bfr.bund.de/de/presseinformation/2012/26/cassia_zimt_mit_hohen_ cumaringehalten_nur_massvoll_verzehren131683.html

[4] Bi X, Lim J, Henry C]. Spices in the management of diabetes mellitus. Food Chem. 2017; 217 : 281-93

[5] Fröhner E. Lehrbuch der Arzneimittellehre für Thierärzte. 4. Aufl. Stuttgart: Enke; 1896

[6] Frohne D, Jensen U. Systematik des Pflanzenreichs. 5. Aufl. Stuttgart: Wissenschaftliche Verlagsgesellschaft; 1998

[7] Henn A. Über die pferdeheilkundliche Handschrift des Joachim Christoph Zachen aus dem 18. Jahrhundert nebst einer Würdigung des Georg Simon Winter von Adlersflügel. Diss. med. vet. Berlin 1999

[8] Hiller K, Melzig FM. Lexikon der Arzneipflanzen und Drogen. 2. Aufl. Heidelberg: Spektrum Akademischer Verlag; 2010

[9] Holmes P. The energetics of western herbs. Vol 1, 3. Ed., Snow Lotus Press, Boulder 1997

[10] Nowak B, Schulz B. Taschenlexikon tropischer Nutzpflanzen und ihrer Früchte. Wiebelsheim: Quelle \& Meyer Verlag; 2009
[11] Schilcher (Hrsg.). Leitfaden Phytotherapie. 5. Aufl. München: Urban \& Fischer; 2016

[12] Sellami M, Louati $\mathrm{H}$ et al. Inhibition of pancreatic lipase and amylase by extracts of different spices and plants. Int J Food Sci Nutr. 2016; 6: $1-8$

[13] Teuscher E. Gewürzdrogen. Stuttgart: Wissenschaftliche Verlagsgesellschaft; 2003

[14] Teuscher E, Melzig MF, Lindequist U. Biogene Arzneimittel. 7. Aufl. Stuttgart: Wissenschaftliche Verlagsgesellschaft; 2012

[15] Wabner D, Beier C (Hrsg.). Aromatherapie. München und Jena: Urban \& Fischer; 2009

[16] Yang WZ, Ametaj BN et al. Cinnamaldehyde in feedlot cattle diets: intake, groth performence, carcass characteristics and blood metabolites. J Anim Sci 2010; 88(3): 1082-92

[17] Zeng Z, Zhang S et al. Essential oil and aromatic plants as feed additives in non-ruminant nutrition: a review. J Anim Sci and Biotechnology 2015; 6: 7

[18] Merck. Datenblatt Trans-Zimtaldehyd; Sicherheitsdatenblatt gemäß Verordnung (EG) Nr. 1907/2006, überarbeitet am 31.08.2015 Fa. Merck

\section{Online zu finden unter}

http://dx.doi.org/10.1055/s-0043-106347

๑ Dr. med. vet. Cäcilia Brendieck-Worm

Talstraße 59

67700 Niederkirchen

E-Mail: cbw@phyto-fokus.de

Geb. 1957, 1976-1981 Studium der Veterinärmedizin in Gießen, 1982-1986 Promotion am Institut für Tierzucht und Haustiergenetik der JLU Gießen, von 1985-2013 Mitglied einer tierärztlichen Praxis für Groß- und Kleintiere in der Pfalz, Zusatzbezeichnung und Weiterbildungsermächtigung Biologische Tiermedizin, Leitung des Arbeitskreises Phytotherapie der GGTM. 